\title{
Generalized projective synchronization of chaotic satellites problem using linear matrix inequality
}

\author{
Yousef Farid • Tahmine Vedadi Moghaddam
}

Received: 28 October 2013 / Revised: 17 March 2014 / Accepted: 22 March 2014 / Published online: 9 April 2014

(C) Springer-Verlag Berlin Heidelberg 2014

\begin{abstract}
In this paper, a systematic design procedure for generalized projective synchronization between two identical chaotic satellites systems based on feedback control theory is proposed. This method is developed based on suitable feedback control, combined with the Lyapunov stability theory and linear matrix inequality formulation as a solution of the optimal problem. Two necessary and sufficient conditions for the asymptotic stability of the error dynamic system are obtained. Compared with the predictive-based control method, the proposed method shows comparable and superior performance. The effectiveness of the obtained results is illustrated by numerical simulations.
\end{abstract}

Keywords Generalized projective synchronization (GPS) . Chaotic satellite systems · Lyapunov stability theory ·

Linear matrix inequality (LMI)

\section{Introduction}

Chaos is a very interesting nonlinear phenomenon. The fundamental characteristic of a chaotic system is its sensitive dependence on initial conditions; which means that a small shift in the initial states can lead to extraordinary perturbation in the system states.

During the past two decades, many chaotic systems have been proposed and their complex behaviors have also been

Y. Farid $(\varangle) \cdot$ T. V. Moghaddam

Advanced Power and Control Systems Lab.,

EE Department, Imam Khomeini International University,

Qazvin, Iran

e-mail: y.farid.e.control@gmail.com

T. V. Moghaddam

e-mail: tahminevedadi@gmail.com widely studied [1-3]. In recent years, many engineering applications of chaotic systems are discussed such as secure communication, digital communication, power electronic devices, power quality, biological systems, chemical reactions analysis and information processing [4-6]. As a special case, in space technology progresses, the need for improved satellite systems by better understanding of satellite dynamics has continuously kept attention [7]. Recently, non-linear dynamics, especially the chaotic attitude dynamics of a satellite have attracted the attention of many scientists [8-10]. For instance it is possible to achieve synchronization among the systems, so that chaos-based communication can be implemented properly on a satellite formulation flying environment [8]. Also both the chaos and optimal control of the steady rotations of a satellite-gyrostat on a circular orbit [10].

The problem of synchronization of a dynamical system is one of the classic topics in engineering science. Synchronization phenomena in chaotic systems have attracted much attention since the work of pecora and carroll [11]. Up to now, various types of synchronization phenomena are being reported for chaotic systems, such as complete synchronization (CS) [12], phase synchronization (PS) [13], anti-phase synchronization (APS) [14], lag synchronization (LS) [15], and generalized synchronization (GS) [16], and projective function synchronization [17].

In 1999, projective synchronization has been first reported by Mainieri and Rehacek [18] in partially linear systems that the master and slave vectors synchronize up to a constant scaling factor ( $\alpha$ proportional relation). CS [12] can be regarded as a special case of projective synchronization characterized at $\alpha=1$, as well as anti-phase synchronization [14] at $\alpha=-1$.

Many methods have been presented for the synchronization of chaotic system such as periodic parametric perturbation method [19], drive-response synchronization method 
(a)

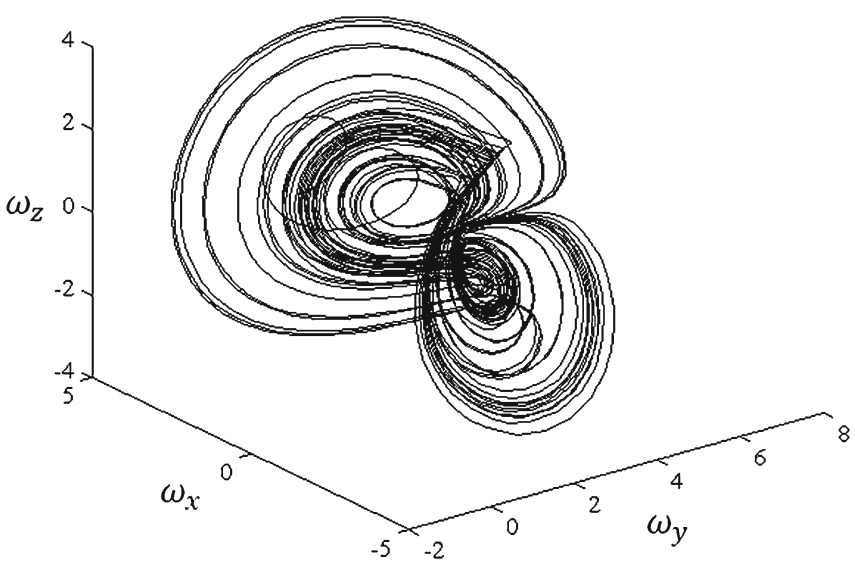

(c)

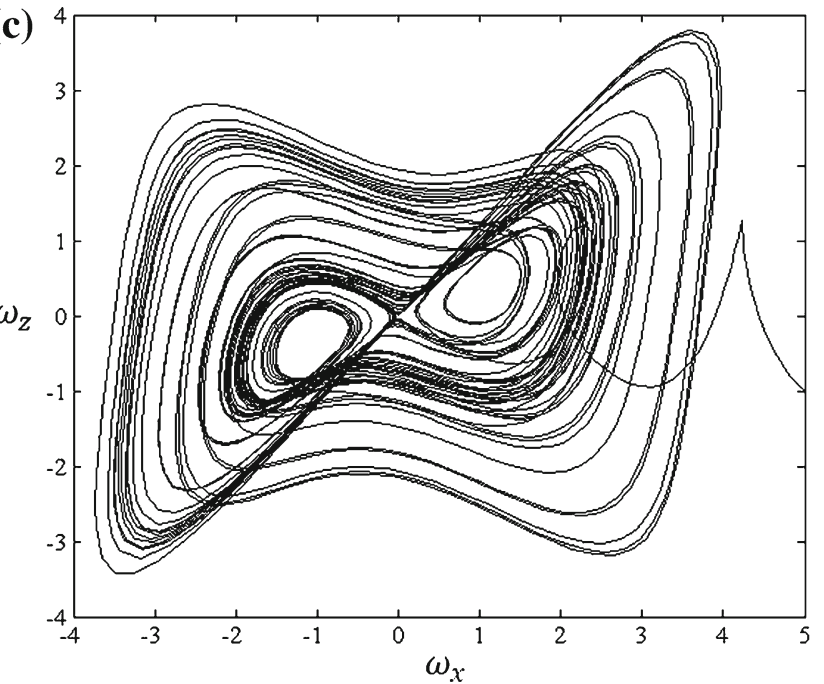

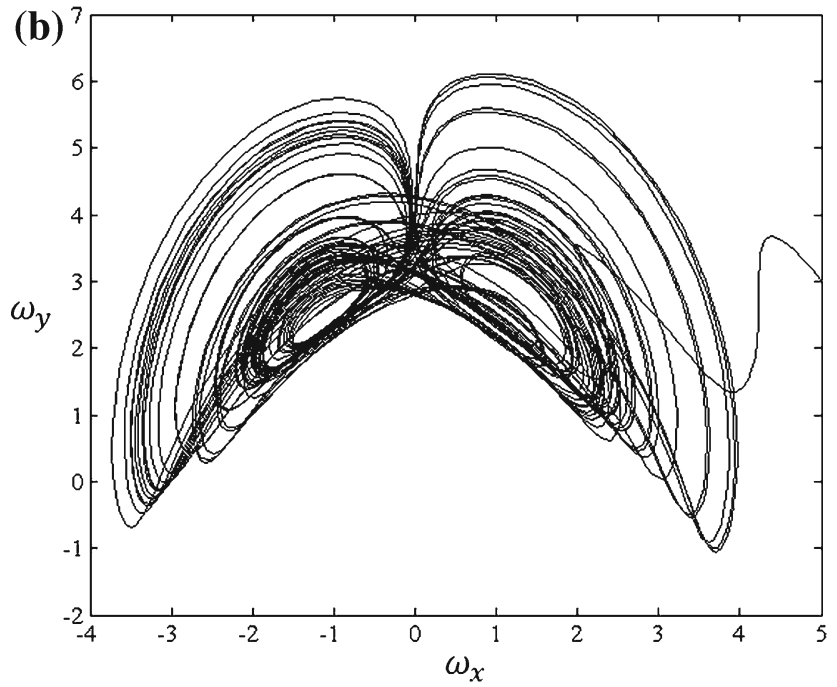

(d)

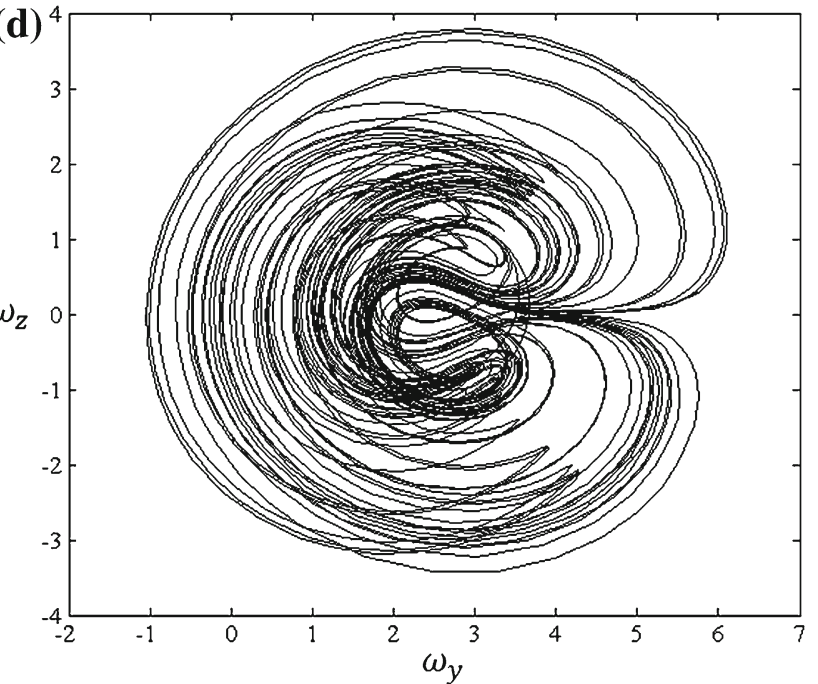

Fig. 1 a Chaotic attractor of satellite system, b trajectories projected on the $\omega_{x}-\omega_{y}$ plane, $\mathbf{c}$ trajectories projected on the $\omega_{x}-\omega_{z}$ plane, d trajectories projected on the $\omega_{y}-\omega_{z}$ plane

[20], active control method [21], adaptive control method [14], sliding mode control method [22], and $H^{\infty}$ control method [23].

The synchronization problem is interpreted as a stabilization one. The goal is to stabilize, at the origin, the discrepancy between the drive and response systems. Discrepancy is defined as the dynamical differences between the drive and response systems. Recently, Sadaoui et al. [24] presented a predictive control strategy for synchronization of chaotic satellites systems.

Most recently, a new synchronization, called generalized projective synchronization, has been observed in the chaotic systems [25-27]. For example nonlinear controller is applied to investigate the generalized projective synchronization for a controlled chaotic gyroscope with a periodic gyroscope dynamical system [25]. Also the generalized projective synchronization of a class of hyperchaotic systems is studied [27].
Linear matrix inequalities (LMIs) have been playing an increasingly important role in the field of optimization and control theory because of the widespread problems (linear and convex quadratic inequalities, matrix norm inequalities, convex constraints etc.) can be written as LMIs [28,29]. In addition, LMI technique for chaos control is concerned; Chen et al. [30] proposed a LMI-based linear-state-feedback control design to achieve the synchronization of uncertain chaotic systems. The control system is transformed into a LMI system whose feedback gain can be directly solved by a LMI solver. Wang et al. [31] with designing a feedback controller based on sliding mode technique investigated the chaos control problem for a general class of chaotic systems, where controller's parameter is obtained from a LMI. And Singh in [32] is presented new criteria for the global robust stability of interval Hopfield-type delayed neural networks.

The aim of this paper is to present a LMI-based feedback controller for the generalized projective synchroniza- 
Fig. 2 State trajectories of the satellites drive and response systems with the scaling factor $\alpha=0.5$
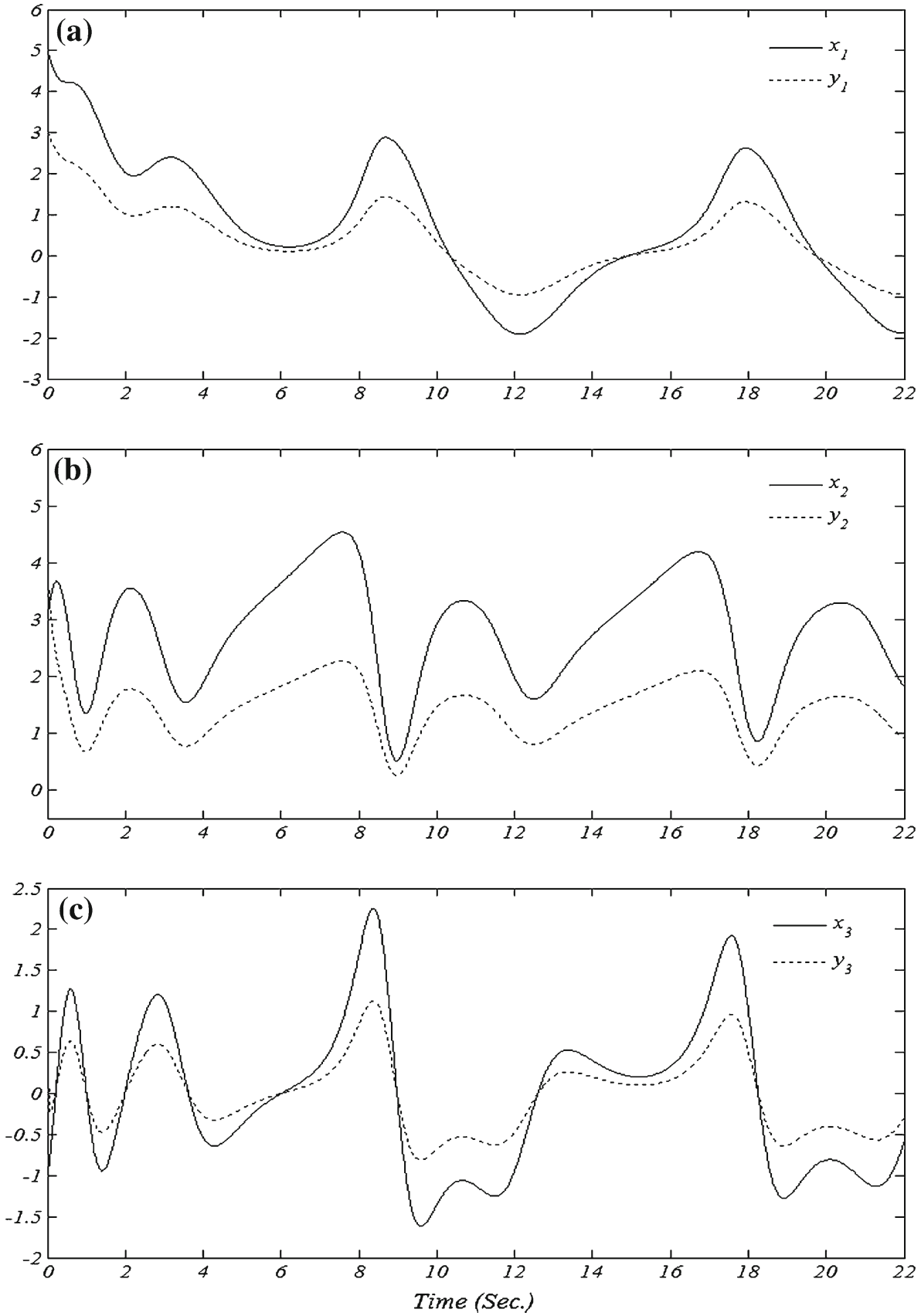

tion between two identical chaotic satellite systems, where the stability of the proposed method is guaranteed by using Lyapunov stability theory. It will be shown that the performance of the proposed scheme is improved compared to a recently published paper [24].

\section{System description and problem formulation}

\subsection{Satellite system with chaotic dynamics}

Recently, the dynamic equations of several spacecraft/satellite systems with the aim of attitude/ congestion control have been studied [33-35]. The dynamical equations of a typical satellite are as [24]:

$$
\left\{\begin{array}{l}
I_{x} \dot{\omega}_{x}=\omega_{y} \omega_{z}\left(I_{y}-I_{z}\right)+h_{x}+u_{x} \\
I_{y} \dot{\omega}_{y}=\omega_{x} \omega_{z}\left(I_{z}-I_{x}\right)+h_{y}+u_{y} \\
I_{z} \dot{\omega}_{z}=\omega_{x} \omega_{y}\left(I_{x}-I_{y}\right)+h_{z}+u_{z}
\end{array}\right.
$$

where $I_{x}, I_{y}$ and $I_{z}$ are the principal moments of inertia; $\omega_{x}, \omega_{y}$ and $\omega_{z}$ are the angular velocities of the satellite; $h_{x}, h_{y}$ and $h_{z}$ are perturbing torques; and $u_{x}, u_{y}$ and $u_{z}$ are the three control torques. Earlier papers [24,36] have taken $I_{x}=3,000 \mathrm{~kg} \mathrm{~m}^{2}, I_{y}=2,000 \mathrm{~kg} \mathrm{~m}^{2}$ and $I_{z}=1,000 \mathrm{~kg} \mathrm{~m}^{2}$ 
Fig. 3 GPS state errors with the scaling factor $\alpha=0.5$

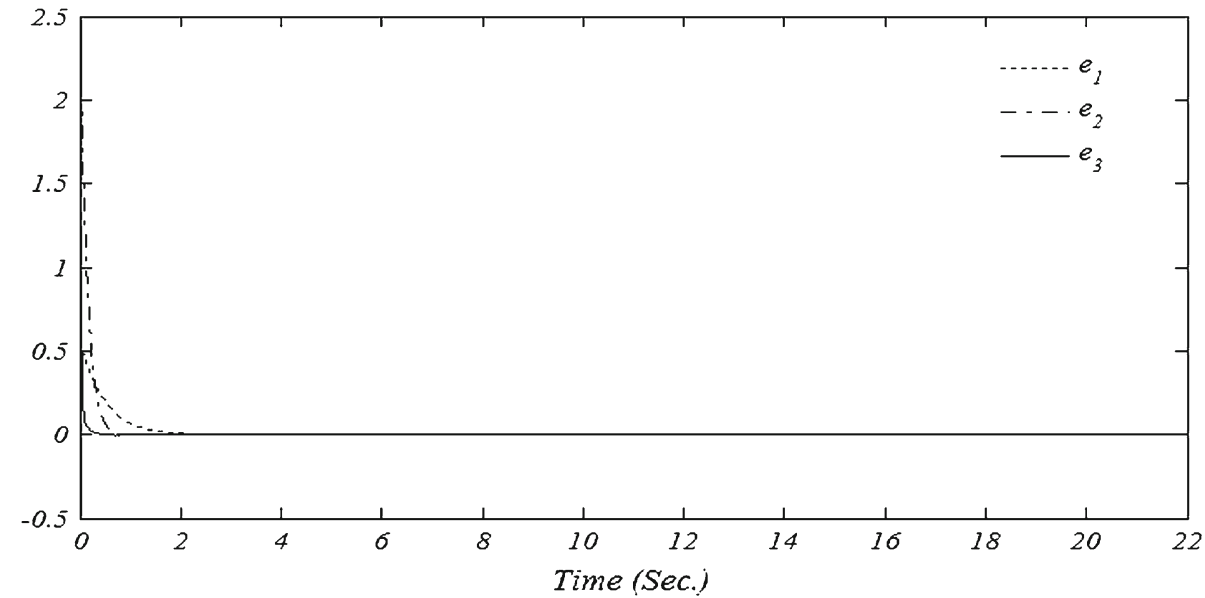

with the perturbing torques defined by:

$$
\left[\begin{array}{l}
h_{x} \\
h_{y} \\
h_{z}
\end{array}\right]=\left[\begin{array}{ccc}
-1200 & 0 & 1000 \sqrt{6} / 2 \\
0 & 350 & 0 \\
-1000 \sqrt{6} & 0 & -400
\end{array}\right]\left[\begin{array}{c}
\omega_{x} \\
\omega_{y} \\
\omega_{z}
\end{array}\right]
$$

These torques are chosen such that the satellite is forced into chaotic motion. The chaotic attractor and chaotic trajectories of the system (1) are shown in Fig. 1a and b-d, respectively.

\subsection{Synchronization problem formulation}

Consider the following two identical satellites systems, where the drive system and response system are denoted with $x$ and $y$, respectively.

\subsubsection{Drive system}

$$
\left\{\begin{array}{l}
\dot{x}_{1}=\frac{1}{3} x_{2} x_{3}-0.4 x_{1}+\frac{\sqrt{6}}{6} x_{3} \\
\dot{x}_{2}=-x_{1} x_{3}+0.175 x_{2} \\
\dot{x}_{3}=x_{1} x_{2}-\sqrt{6} x_{1}-0.4 x_{3}
\end{array}\right.
$$

where $x_{i}(i=1,2,3)$ is drive system state.

\subsubsection{Response system}

$$
\left\{\begin{array}{l}
\dot{y}_{1}=\frac{1}{3} y_{2} y_{3}-0.4 y_{1}+\frac{\sqrt{6}}{6} y_{3}+u_{1} \\
\dot{y}_{2}=-y_{1} y_{3}+0.175 y_{2}+u_{2} \\
\dot{y}_{3}=y_{1} y_{2}-\sqrt{6} y_{1}-0.4 y_{3}+u_{3}
\end{array}\right.
$$

where $y_{i} \quad(i=1,2,3)$ is response system state.

Definition 1 The projective synchronization error between drive system and response system is defined as $e_{i}=y_{i}-$ $\alpha x_{i} ; i=1,2,3$, where $\alpha \neq 0$ is called a scaling factor.

The objective of this study is to propose an approach to designing a suitable controller $u_{i}(t) ; i=1,2,3$ such that

$$
\begin{aligned}
& \lim _{t \rightarrow \infty}\left\|e_{i}(t)\right\|=\lim _{t \rightarrow \infty}\left\|y_{i}(t)-\alpha x_{i}(t)\right\|=0 \\
& \quad i=1,2,3
\end{aligned}
$$

where $\|\cdot\|$ denotes the Euclidean norm of a vector. If Eq. (5) is satisfied, then it can be said that systems (3) and (4) have obtained projective synchronization.

According to the Definition 1, the error dynamic system can be obtained from (3) and (4) as follows:

$\left\{\begin{aligned} \dot{e}_{1}= & \frac{1}{3}\left(y_{2} y_{3}-\alpha x_{2} x_{3}\right)-0.4\left(y_{1}-\alpha x_{1}\right)+\frac{\sqrt{6}}{6} \\ & \times\left(y_{3}-\alpha x_{3}\right)+u_{1} \\ \dot{e}_{2}= & -\left(y_{1} y_{3}-\alpha x_{1} x_{3}\right)+0.175\left(y_{2}-\alpha x_{2}\right)+u_{2} \\ \dot{e}_{3}= & \left(y_{1} y_{2}-\alpha x_{1} x_{2}\right)-\sqrt{6}\left(y_{1}-\alpha x_{1}\right)-0.4\left(y_{3}-\alpha x_{3}\right)+u_{3}\end{aligned}\right.$

Base on Definition 1, it is directly obtained that

$\left\{\begin{array}{l}y_{2} y_{3}-\alpha x_{2} x_{3}=\alpha x_{3} e_{2}+\alpha x_{2} e_{3}+e_{2} e_{3}+\alpha(\alpha-1) x_{2} x_{3} \\ y_{1} y_{3}-\alpha x_{1} x_{3}=\alpha x_{3} e_{1}+\alpha x_{1} e_{3}+e_{1} e_{3}+\alpha(\alpha-1) x_{1} x_{3} \\ y_{1} y_{2}-\alpha x_{1} x_{2}=\alpha x_{2} e_{1}+\alpha x_{1} e_{2}+e_{1} e_{2}+\alpha(\alpha-1) x_{1} x_{2}\end{array}\right.$

With substituting Eq. (7) into Eq. (6), it yields:

$\left\{\begin{aligned} \dot{e}_{1}= & -0.4 e_{1}+\frac{1}{3} \alpha x_{3} e_{2}+\left(\frac{\sqrt{6}}{6}+\frac{1}{3} \alpha x_{2}\right) e_{3}+\frac{1}{3} e_{2} e_{3} \\ & +\frac{1}{3} \alpha(\alpha-1) x_{2} x_{3}+u_{1} \\ \dot{e}_{2}= & -\alpha x_{3} e_{1}+0.175 e_{2}-\alpha x_{1} e_{3}-e_{1} e_{3}-\alpha(\alpha-1) x_{1} x_{3}+u_{2} \\ \dot{e}_{3}= & \left(\alpha x_{2}-\sqrt{6}\right) e_{1}+\alpha x_{1} e_{2}-0.4 e_{3}+e_{1} e_{2}+\alpha(\alpha-1) x_{1} x_{2}+u_{3}\end{aligned}\right.$

where $u_{i}(i=1,2,3)$ are control inputs that will be designed by LMI-based feedback control method.

\section{LMI-based feedback controller design and it's stability analysis}

The LMI-based feedback controller is a combination of two design procedure: the feedback controller design and the LMI relation formulation, which are given, respectively. 
Fig. 4 State trajectories of the satellites drive and response systems with the scaling factor $\alpha=-1$
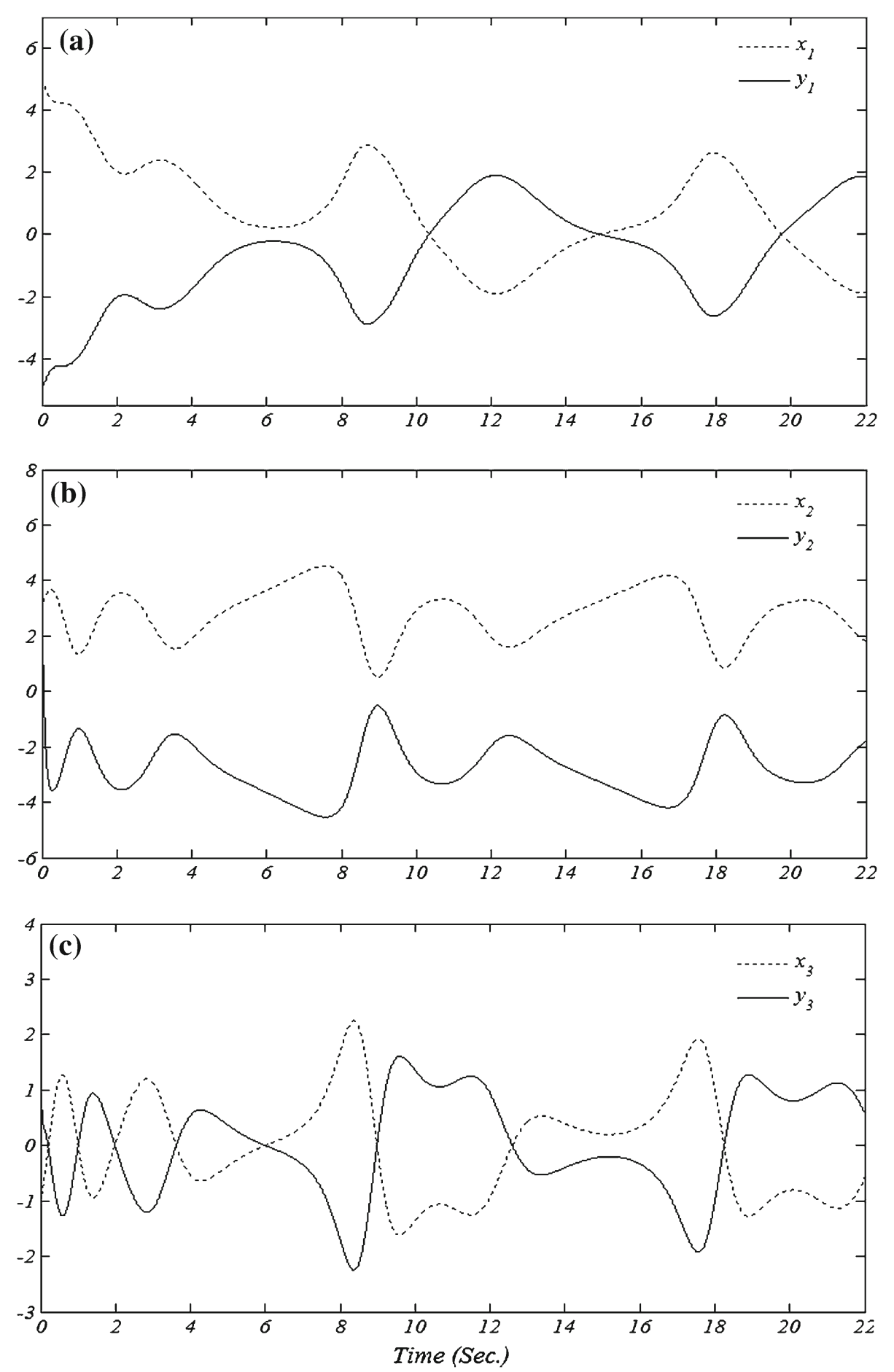

For the error dynamic system, Eq. (8), to design the controller, set the control law as:

$$
\left\{\begin{array}{l}
u_{1}=-\frac{1}{3} \frac{(\alpha-1)}{\alpha}\left(y_{2}-e_{2}\right)\left(y_{3}-e_{3}\right)-\gamma_{1} e_{1} \\
u_{2}=\frac{(\alpha-1)}{\alpha}\left(y_{1}-e_{1}\right)\left(y_{3}-e_{3}\right)-\gamma_{2} e_{2} \\
u_{3}=-\frac{(\alpha-1)}{\alpha}\left(y_{1}-e_{1}\right)\left(y_{2}-e_{2}\right)-\gamma_{3} e_{3}
\end{array}\right.
$$

where $\gamma_{i}(i=1,2,3)$ are positive feedback gains that will be obtained by LMI.

For the error dynamic system in Eq. (8), the existence of a quadratic Lyapunov function $V(t)=e^{T}(t) P e(t), P>$ $0, P \in R^{3 \times 3}$ is a necessary and sufficient condition for asymptotic stability [37]. Define a Lyapunov function candidate of the Eq. (8) as follows:

$V(t)=\rho_{1} e_{1}^{2}(t)+\rho_{2} e_{2}^{2}(t)+\rho_{3} e_{3}^{2}(t)$ 
Fig. 5 GPS state errors with the scaling factor $\alpha=-1$

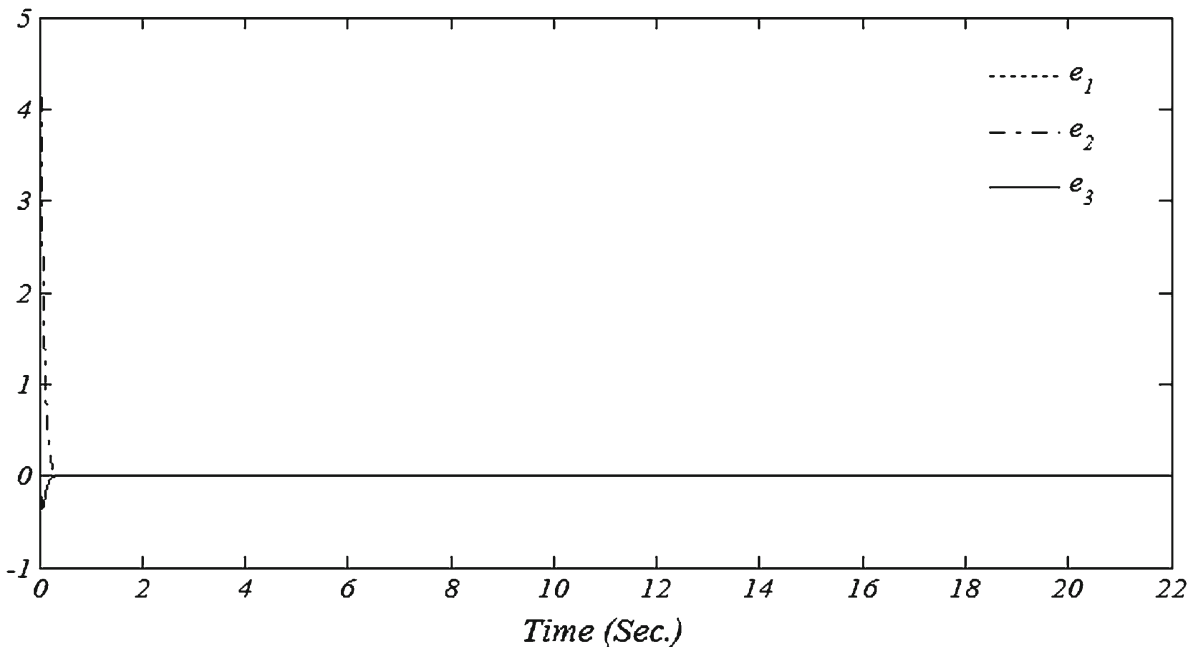

where $\rho_{i}(i=1,2,3)$ are positive constants and therefore this Lyapunov function is positive definite. Then, taking the derivative of Eq. (10) with respect to time, and with applying the controller (9) in to error dynamic Eq. (8), the result will be as follows:

$$
\begin{aligned}
\dot{V}= & 2 \rho_{1} e_{1} \dot{e}_{1}+2 \rho_{2} e_{2} \dot{e}_{2}+2 \rho_{3} e_{3} \dot{e}_{3} \\
= & -2\left(0.4+\gamma_{1}\right) \rho_{1} e_{1}^{2}-2\left(-0.175+\gamma_{2}\right) \rho_{2} e_{2}^{2} \\
& -2\left(0.4+\gamma_{3}\right) \rho_{3} e_{3}^{2}+2\left(\frac{1}{3} \rho_{1}-\rho_{2}\right) \alpha x_{3} e_{1} e_{2} \\
& +2\left(\left(\frac{\sqrt{6}}{6}+\frac{1}{3} \alpha x_{2}\right) \rho_{1}+\left(\alpha x_{2}-\sqrt{6}\right) \rho_{3}\right) e_{1} e_{3} \\
& +2\left[-\rho_{2}+\rho_{3}\right] \alpha x_{1} e_{2} e_{3}+2\left[\frac{1}{3} \rho_{1}-\rho_{2}+\rho_{3}\right] e_{1} e_{2} e_{3}
\end{aligned}
$$

If the relation between Lyapunov function's parameters are chosen as

$\rho_{1}=3\left(\rho_{2}-\rho_{3}\right)$ the following result can be obtained

$$
\begin{aligned}
\dot{V}(t)= & -6\left(0.4+\gamma_{1}\right)\left(\rho_{2}-\rho_{3}\right) e_{1}^{2} \\
& -2\left(-0.175+\gamma_{2}\right) \rho_{2} e_{2}^{2} \\
& -2\left(0.4+\gamma_{3}\right) \rho_{3} e_{3}^{2}-2 \rho_{3} \alpha x_{3} e_{1} e_{2} \\
& +2\left(\left(\frac{\sqrt{6}}{2}+\alpha x_{2}\right) \rho_{2}-\frac{3 \sqrt{6}}{2} \rho_{3}\right) e_{1} e_{3} \\
& +2\left(\rho_{3}-\rho_{2}\right) \alpha x_{1} e_{2} e_{3} \leq-6\left(4+\gamma_{1}\right)\left(\rho_{2}-\rho_{3}\right) e_{1}^{2} \\
& -2\left(-0.175+\gamma_{2}\right) \rho_{2} e_{2}^{2}-2\left(0.4+\gamma_{3}\right) \rho_{3} e_{3}^{2} \\
& +2 \rho_{3}|\alpha|\left|x_{3}\right|\left|e_{1}\right|\left|e_{2}\right|+2\left(\left(\frac{\sqrt{6}}{2}+|\alpha|\left|x_{2}\right|\right) \rho_{2}\right. \\
& \left.+\frac{3 \sqrt{6}}{2} \rho_{3}\right)\left|e_{1}\right|\left|e_{3}\right|+2\left(\rho_{3}+\rho_{2}\right)|\alpha|\left|x_{1}\right|\left|e_{2}\right|\left|e_{3}\right|
\end{aligned}
$$

In LMI relation formulation, it can be said that because the trajectories of a drive chaotic system are all bounded, there exist three positive constants $M_{1}, M_{2}$ and $M_{3}$ such that $\left|x_{1}\right| \leq M_{1},\left|x_{2}\right| \leq M_{2}$ and $\left|x_{3}\right| \leq M_{3}$ and so $\dot{v} \leq$ $\left(\left|e_{1}\right|,\left|e_{2}\right|,\left|e_{3}\right|\right) \Omega\left(\left|e_{1}\right|,\left|e_{2}\right|,\left|e_{3}\right|\right)^{T}$, where

$\Omega=\left[\begin{array}{ccc}-6\left(0.4+\gamma_{1}\right)\left(\rho_{2}-\rho_{3}\right) & \rho_{3}|\alpha| M_{3} & \left(\left(\frac{\sqrt{6}}{2}+|\alpha| M_{2}\right) \rho_{2}+\frac{3 \sqrt{6}}{2} \rho_{3}\right) \\ * & -2\left(-0.175+\gamma_{2}\right) \rho_{2} & \left(\rho_{2}+\rho_{3}\right)|\alpha| M_{1} \\ * & * & -2\left(0.4+\gamma_{3}\right) \rho_{3}\end{array}\right]$ 
Fig. 6 State trajectories of the satellites drive and response systems with the scaling factor $\alpha=2$
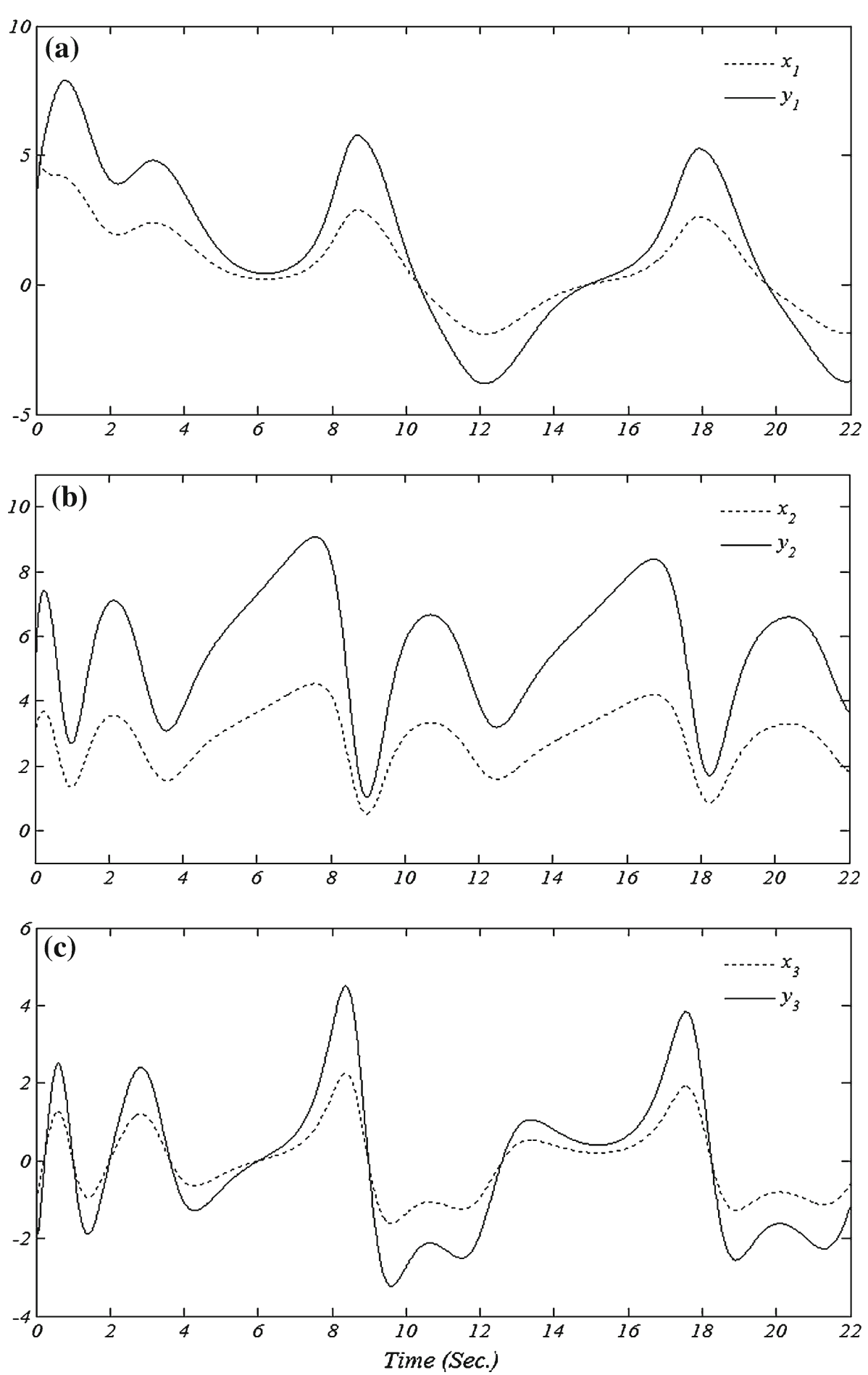

Note that $\Omega$ is a symmetrical matrix where $*$ denotes the symmetric terms. To guarantee that the synchronous errors converge to zero, the matrix $\Omega$ must be negative definite.
Thus, the condition $\Omega<0$ is a sufficient condition for the projective synchronization of the satellite chaotic systems. The condition $\Omega<0$ and $\rho_{1}, \rho_{2}, \rho_{3}>0$ can be expressed as 
Fig. 7 GPS state errors with the scaling factor $\alpha=2$
Fig. 8 GPS state errors for stabilization of the systems to the unstable fixed point $(0,0,0)$
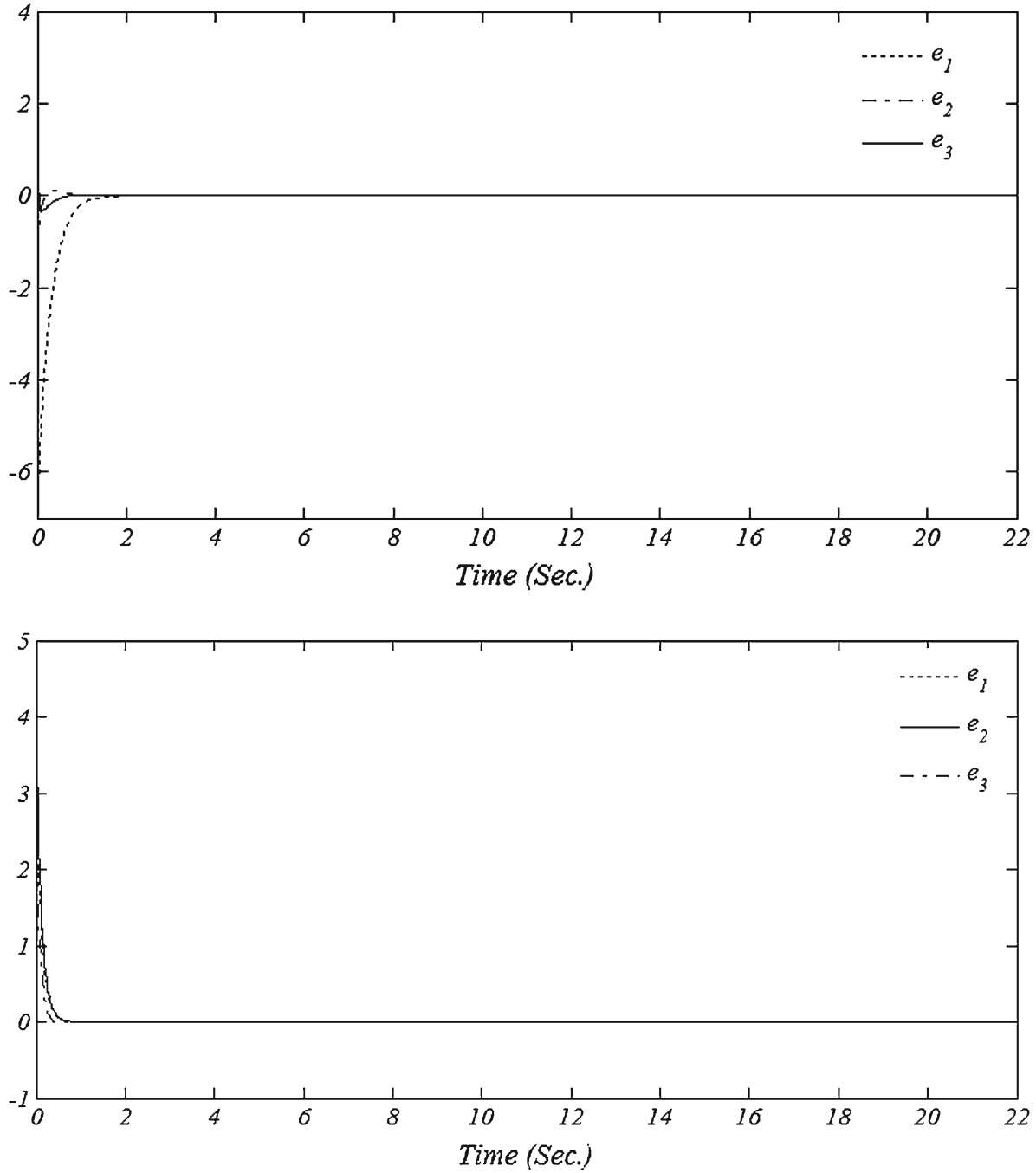

Time (Sec.)

$$
\begin{aligned}
& {\left[\begin{array}{ccc}
-6\left(0.4+\gamma_{1}\right)\left(\rho_{2}-\rho_{3}\right) & \rho_{3}|\alpha| M_{3} & \left(\left(\frac{\sqrt{6}}{2}+|\alpha| M_{2}\right) \rho_{2}+\frac{3 \sqrt{6}}{2} \rho_{3}\right) \\
* & -2\left(-0.175+\gamma_{2}\right) \rho_{2} & \left(\rho_{2}+\rho_{3}\right)|\alpha| M_{1} \\
* & * & -2\left(0.4+\gamma_{3}\right) \rho_{3}
\end{array}\right]<0} \\
& {\left[\begin{array}{ccc}
3\left(\rho_{3}-\rho_{2}\right) & 0 & 0 \\
0 & -\rho_{2} & 0 \\
0 & 0 & -\rho_{3}
\end{array}\right]<0}
\end{aligned}
$$

Which are LMIs in $\gamma_{1}, \gamma_{2}, \gamma_{3}, \rho_{2}$ and $\rho_{3}$, and it can be solved directly by various LMI solvers such as MATLAB's LMI Control Toolbox. Based on Lyapunov's second method for stability, the system is asymptotically stable.

Therefore, if the conditions (15a) and (15b) are satisfied, the error functions $e_{i}(i=1,2,3)$ converges to zero as the time $t$ tends to infinity, and projective synchronization between systems (3) and (4) is achieved under the controller (9).

\section{Simulation}

In this section, computer simulations are used to verify and demonstrate the effectiveness of the above presented LMIbased control method. The simulation results are carried out using MATLAB software. The fourth order Runge-Kutta integration algorithm was performed to solve the differential equations. 
Table 1 Comparison between two different methods of stabilization.

\begin{tabular}{lll}
\hline Criteria & SSSE & SEST (Sec.) \\
\hline $\begin{array}{ll}\text { Predictive-based control method }[24] \\
\text { Proposed method }\end{array}$ & $\operatorname{SSSE}\left(e_{1}\right)=248.94, \operatorname{SSSE}\left(e_{2}\right)=260.54, \operatorname{SSSE}\left(e_{3}\right)=90.75$ & $\approx 15.20$ \\
& $\operatorname{SSSE}\left(e_{1}\right)=36.22, \operatorname{SSSE}\left(e_{2}\right)=65.41, \operatorname{SSSE}\left(e_{3}\right)=24.46$ & $\approx 0.8764$ \\
\hline
\end{tabular}

For simulation with the scaling factor $\alpha=0.5$, the design parameters are taken as $\gamma_{1}=2.2669, \gamma_{2}=3.7156$, and $\gamma_{3}=14.5751$. For simulation with the scaling factor $\alpha=-1$ (APS), the design parameters are taken as $\gamma_{1}=4.5378, \gamma_{2}=8.2017$, and $\gamma_{3}=21.6570$. For simulation with the scaling factor $\alpha=2$, the design parameters are taken as $\gamma_{1}=10.9989, \gamma_{2}=16.5075$, and $\gamma_{3}=34.5318$. As it is said before all these parameters can be obtained directly by solving Eq. (15) in MATLAB's LMI Control Toolbox. The initial values of satellite systems are taken as $x_{1}=5, x_{2}=3, x_{3}=-1$ and $y_{1}=3, y_{2}=4.1, y_{3}=2$.

The simulation results with the scaling factor $\alpha=0.5$ are shown in Figs. 2 and 3. The simulation results with the scaling factor $\alpha=-1$ (APS) are shown in Figs. 4 and 5. The simulation results with the scaling factor $\alpha=2$ are shown in Figs. 6 and 7. From Figs. 3, 5, and 7, it is clear that for an initial error between drive and response systems, after a finite period of time, the initial error will converge to zero asymptotically and GPS between drive and response systems will be achieved.

To present a quantitative comparison between the proposed method and Sadaoui method [24] in stabilization of unstable fixed point $\left[\begin{array}{lll}0 & 0 & 0\end{array}\right]^{T}$, two following criteria are used:

(a) Synchronization error settling time (SEST). It is the time at which $\|e\|<0.005$.

(b) Sum of squared synchronization error (SSSE) up to SEST.

The simulation result for synchronization of unstable fixed point $\left[\begin{array}{lll}0 & 0 & 0\end{array}\right]^{T}$ is shown in Fig. 8. A comparative result between proposed method and Sadaoui method [24] is given in Table 1. As it is known, the less the SEST, the convergence is sooner. With the less the SSSE the better stabilization is achieved. Referring to the Table 1, it can be concluded that the speed of synchronization with the proposed method is better than that with predictive-based control method.

Because the anti-synchronization $(\alpha=-1)$, and projective synchronization $(\alpha=0.5,2)$ are included in generalized projective synchronization, this paper results are applicable for different $\alpha$ scales (full range GPS). How to realize the generalized projective synchronization of two uncertain and noisy chaotic satellite systems will be presented in our future work.

\section{Conclusion}

In this paper, Lyapunov stability theory and LMI technique have been employed to obtain optimal solution for generalized projective synchronization of the chaotic satellite systems. It extends the control capability to achieve a full range GPS of all state variables in a feedback way. This method had two advantages in comparison with other result in the same field. It was illustrated that the speed of synchronization of the states was very fast and it was better than that with predictive-based control methodology. The errors had been greatly reduced and had converged very rapidly to zero. Numerical simulations were illustrated to demonstrate the effectiveness of the proposed approach.

\section{References}

1. Vicha T, Dohnal M (2008) Qualitative feature extractions of chaotic systems. Chaos Solitons Fract 38:364-373

2. Chen Z, Zhang X, Bi Q (2008) Bifurcations and chaos of coupled electrical circuits. Nonlinear Anal: Real World Appl 9:1158-1168

3. Torkamani S, Butcher EA (2013) Delay, state, and parameter estimation in chaotic and hyperchaotic delayed systems with uncertainty and time-varying delay. Int J Dyn Control 1(2):135-163

4. Arman KB, Kia F, Naser P, Henry L (2009) A chaotic secure communication scheme using fractional chaotic systems based on an extended fractional Kalman filter. Commun Nonlinear Sci Numer Simul 14:863-879

5. El-Gohary A (2009) Chaos and optimal control of equilibrium states of tumor system with drug. Chaos Solitons Fract 41:425435

6. Huang CF, Cheng KH, Yan JJ (2009) Robust chaos synchronization of four-dimensional energy resource systems subject to unmatched uncertainties. Commun Nonlinear Sci Numer Simul 14:2784-2792

7. Kuang J, Leung AYT, Tan S (2003) Hamiltonian and chaotic attitude dynamics of an orbiting gyrostat satellite under gravitygradient torques. Physica D 186:1-19

8. Grzybowski JMV, Rafikov M, Macau EEN (2010) Synchronization analysis for chaotic communication on a satellite formulation flying. Acta Astronaut 67(7-8):881-891

9. Aslanov VS, Doroshin AV (2010) Chaotic dynamics of an unbalanced gyrostat. J Appl Math Mech 74(5):524-535

10. Gohary AE (2009) Chaos and optimal control of steady-state rotation of a satellite-gyrostat on a circular orbit. Chaos Solitons Fract 42(5):2842-2851

11. Pecora LM, Carroll TL (1990) Synchronization in chaotic systems. Phys Rev Lett 64:821-824

12. Huang LL, Feng RP, Wang M (2004) Synchronization of chaotic systems via nonlinear control. Phys Lett A 320:271-275

13. Banerjee S, Saha P, Chowdhury AR (2004) On the application of adaptive control and phase synchronization in non-linear fluid dynamics. Int J Non-Linear Mech 39:25-31 
14. Li R, Xu W, Li S (2009) Anti-synchronization on autonomous and non-autonomous chaotic systems via adaptive feedback control. Chaos Solitons Fract 40:1288-1296

15. Ge ZM, Wong YT, Li SY (2008) Temporary lag and anticipated synchronization and anti-synchronization of uncoupled timedelayed chaotic systems. J Sound Vib 318:267-278

16. Gang Z, Zengrong L, Zhongjun M (2007) Generalized synchronization of different dimensional chaotic dynamical systems. Chaos Solitons Fract 32:773-779

17. Fuhong M, Albert CJ (2013) On the projective function synchronization of chaos for two gyroscope systems under sinusoidal constraints. Int J Dyn Control 1(3):203-213

18. Mainieri R, Rehacek J (1999) Projective synchronization in threedimensional chaotic systems. Phys Rev Lett 82:3042-3045

19. Blazejczyk-Okolewska B, Brindley J, Czolczynski K, Kapitaniak $\mathrm{T}$ (2001) Antiphase synchronization of chaos by noncontinuous coupling: two impacting oscillators. Chaos Solitons Fract 12:18231826

20. Yang XS, Duan CK, Liao XX (1999) A note on mathematical aspects of drive-response type synchronization. Chaos Solitons Fract 10:1457-1462

21. Feng CF (2010) Projective synchronization between two different time-delayed chaotic systems using active control approach. Nonlinear Dyn 62:453-459

22. Kuo CL (2011) Design of a fuzzy sliding-mode synchronization controller for two different chaos systems. Comput Math Appl 61:2090-2095

23. Ahn CK, Jung ST, Kang SK, Joo SC (2010) Adaptive $H^{\infty}$ synchronization for uncertain chaotic systems with external disturbance. Commun Nonlinear Sci Numer Simul 15:2168-2177

24. Sadaoui D, Boukabou A, Merabtine N, Benslama M (2011) Predictive synchronization of chaotic satellites systems. Expert Syst Appl 38:9041-9045

25. Min F (2012) Analysis of generalized projective synchronization for a chaotic gyroscope with a periodic gyroscope. Commun Nonlinear Sci Numer Simul 17(12):4917-4929
26. Wu X, Wang H, Lu H (2012) Modified generalized projective synchronization of a new fractional-order hyperchaotic system and its application to secure communication. Nonlinear Anal: Real World Appl 13(3):1441-1450

27. Wang XY, Fan B (2012) Generalized projective synchronization of a class of hyperchaotic systems based on state observer. Commun Nonlinear Sci Numer Simul 17(2):953-963

28. Khayati K, Bigras P, Dessaint LA (2009) LuGre model-based friction compensation and positioning control for a pneumatic actuator using multi-objective output-feedback control via LMI optimization. Mechatronics 19:535-547

29. Ho WH, Chen SH, Liu TK, Chou JH (2010) Design of robustoptimal output feedback controllers for linear uncertain systems using LMI-based approach and genetic algorithm. Informulation Sci 180:4529-4542

30. Chen F, Zhang W (2007) LMI criteria for robust chaos synchronization of a class of chaotic systems. Nonlinear Anal 67:3384-3393

31. Wang H, Han Z, Xie Q, Zhang W (2009) Sliding mode control for chaotic systems based on LMI. Commun Nonlinear Sci Numer Simul 14:1410-1417

32. Singh V (2010) New LMI-based criteria for global robust stability of delayed neural networks. Appl Math Modell 34:2958-2965

33. Qinglei H, Youmin Z, Xing H, Bing X (2011) Adaptive Integraltype sliding mode control for spacecraft attitude maneuvering under actuator stuck failures. Chin J Aeronaut 24:32-45

34. Varma S, Kumar KD (2010) Fault tolerant satellite attitude control using solar radiation pressure based on nonlinear adaptive sliding mode. Acta Astronaut 66:486-500

35. Nagi F, Ahmed SK, ZainulAbidin AA, Nordin FH (2010) Fuzzy bang-bang relay controller for satellite attitude control system. Fuzzy Sets Syst 161:2104-2125

36. Kemih K, Kemiha A, Ghanes M (2009) Chaotic attitude control of satellite using impulsive control. Chaos Solitons Fract 42:735-744

37. Sabatier J, Moze M, Farges C (2010) LMI stability conditions for fractional order systems. Comput Math Appl 59(5):1594-1609 